\title{
Neural Network Using for Extracting Hidden Information in Images
}

\section{Safwan Omar Hasoon}

Dr.safwan1971@yahoo.com

College of Computer Sciences and Mathematics

University of Mosul, Mosul, Iraq

Received on: 20/03/2012

Accepted on: 28/06/2012

\begin{abstract}
Steganography technique widely spread and varied. With the widening in using steganography, its misuse alarmists arisen. Thus steganalysis comes into sight to deter unwanted secret communications.

In this paper a new scheme proposed for extracting hidden information, this scheme relies on the capability of artificial neural networks for prediction to estimate the original values of the pixels which values of some of them were changed by the affection of data embedding process, and then the present pixel values will be compared with estimated values to identify the embedded data. Multilayer Perceptron MLP neural network used in this scheme to estimate the pixel's original value using its neighbor pixels. The proposed schemes programmed using Matlab v. 7.10.0.499. The proposed schemes has been trained and tested using a data base prepared for this purpose. Then its performance compared with another work in the same field applied in similar conditions. The results showed that the proposed scheme has the ability to achieving the desired with a good rate of success.
\end{abstract}

Keywords: Steganography, Stegnoanalysis, Neural Network.

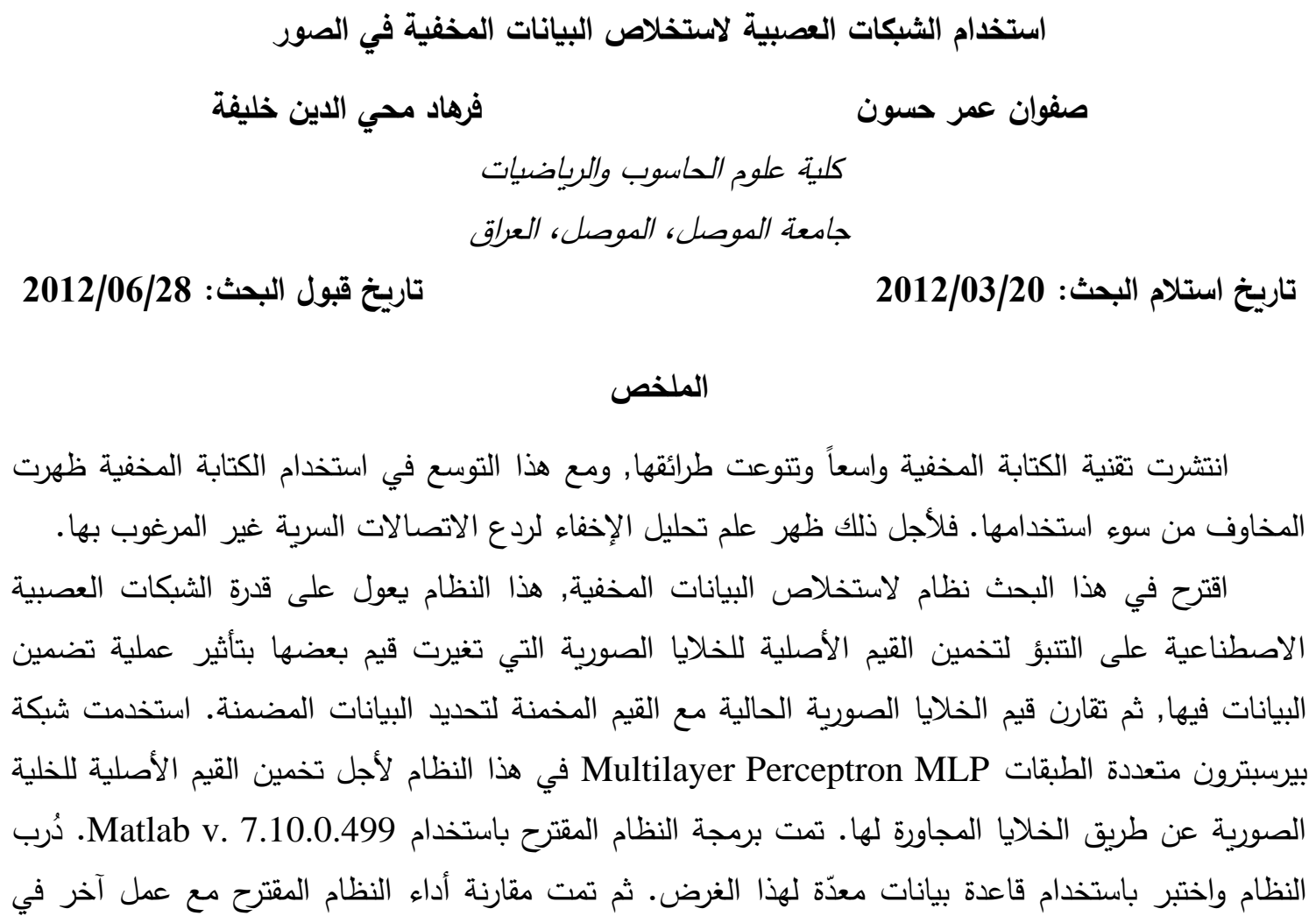


مجال نفسه مطبقاً في ظروف متثابهة. أظهرت النتائج أن النظام له القدرة على إنجاز المطلوب منه بنسبة جيدة من النجاح. الكلمات المفتاحية: الكتابة المخفية، تحليل الاخفاء، الثبكة العصبية.

1 - 1 - 1 - 1

نظراً للتطور الهائل في مجال التقنية الرقية وشبكة الانترنيت والاتصالات أصبحت الخصوصية الشخصية عرضة للانتهاك بسهولة أكبر من ذي قبل. فكان لابد من طرائق تُحفظ بها سرية البيانات الثخصية حال تناقلها لمنع المتطفلين من الاطلاع عليها. لهذا لغرض أوجدت تقنية الكتابة المخفية الرقمية. الكتابة المخفية Steganography هي فن وعلم إخفاء بيانات سرية مهمة في ناقل غير مؤذٍ على نحو

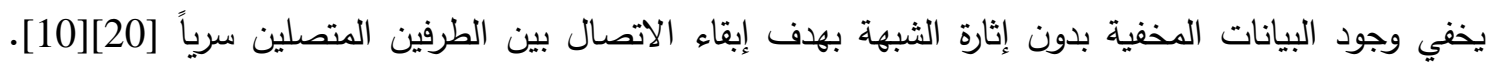

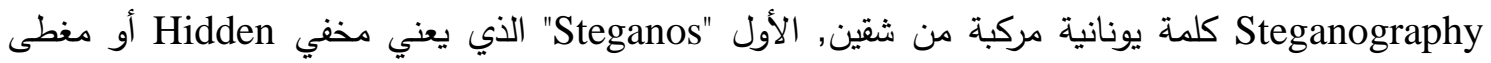

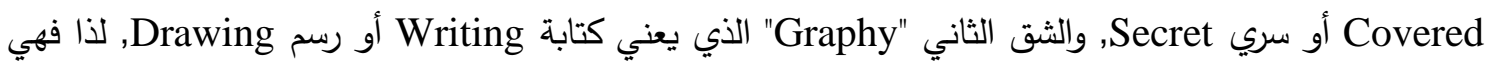

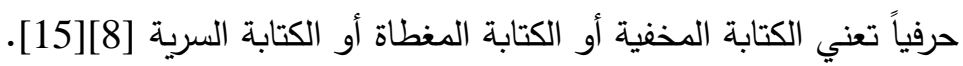
إخفاء المعلومات داخل الصور تقنية واسعة الانتشار في الوقت الحاضر. فالصنه الصور الرقمية لها درجة كبيرة من التكرار غير المفيد في التمثيل ولها تطبيقات واسعة الانتشار في الحياة اليومية. إذ إن الصور الحاملة للرسائل السرية يمكن أن تنتشر بسهولة عبر الانترنيت أو المجاميع الإخبارية. وبالتالي فهي مغرية لإخفاء الإسياء المعلومات.

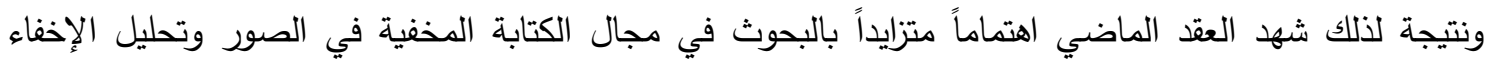
للصور [10][12].

شهدت بدايات العقد الأخير من القرن الماضي ظهور طليعة البحوث حول الكتابة المخفية الرقمية, على الرغم من أن الكتابة المخفية عموماً لها جذور موغلة في عمق التاريخ , إذ استخدمت طرائق مختلفة لانجاز الكتابة

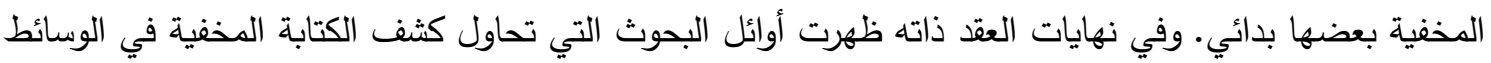

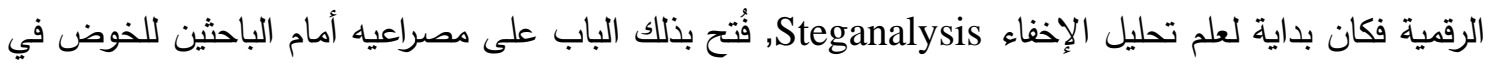

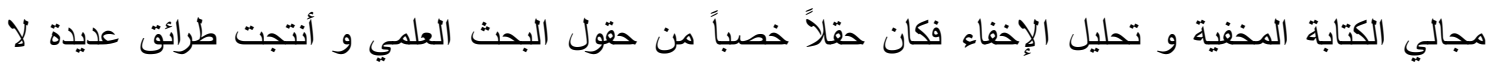
حصر لها في كلا المجالين.

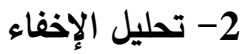

على النقيض من الهدف من إخفاء المعلومات Information Hiding, فإن تحليل الإخفاء Steganalysis

اكتسب تحليل الإخفاء أهمية في حقلي الأمن القومي والعلوم العدلية, لكون كثف الرسائل المخفية يمكن أن يؤدي إلى منع الحوادث الأمنية الكارثية. إن حقل تحليل الإخفاء حقل صعب الإبل جداً بسبب ندرة المعرفة بثأن الخصائص المعينة لوسائط الغطاء (ملفات الصور والصوت والفيديو) التي يمكن أن تستغل لإخفاء المعلومات أو أو التئل اكتثافها على حد سواء. والأساليب المعتمدة في تحليل الإخفاء تعتمد في بعض الأحيان على أساس الخوارزميات المستخدمة في الإخفاء [11]. 
إن أتمتة كثف الرسائل المخفية ضرورة لابد منها لأن الكمية الهائلة من بيانات الصور المخزونة على

أجهزة الحاسوب أو في مواقع الانترنيت تجعل من المستحيل على شخص أن يتحرّى كل صورة على انفراد [4]. تقسم خوارزميات تحليل الإخفاء للصورة حسب خوارزميات الإخفاء التي تعمل عليها إلى نوعين: الأسلوب إنى

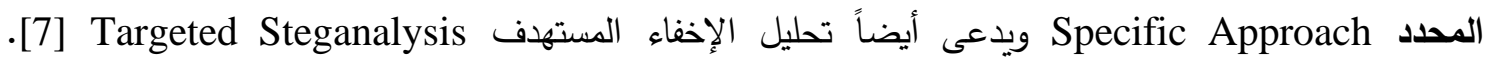
الأسلوب المحدد يمثل فئة تقنيات تحليل الإخفاء التي تعتمد كثيراً على أساس خوارزمية الإخفاء المستخدمة, هذاء الإفاء الأسلوب له نسبة نجاح عالية لاكتثاف وجود الرسالة السرية إذا كانت الرسالة مخفية باستخدام خوارزمية الإخفاء

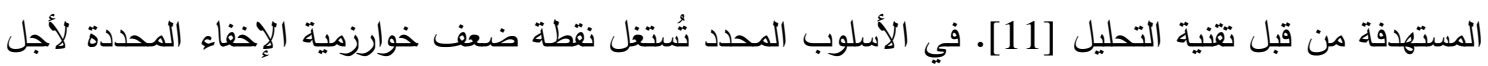
التحليل [10]. في هذا الأسلوب يتم التركيز على الاختلافات التي تحدثها خوارزمية إخفاء معينة في ملف الغطاء

$$
\text { بحيث تعطي مؤشراً على وجود رسالة مخفية. }
$$

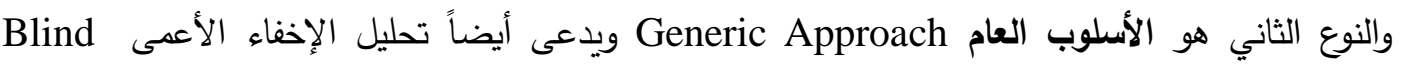
Steganalysis [7]. وهو يمثل فئة تقنيات تحليل الإخفاء التي لا تتطلب معلومات مسبقة حول خوارزميات

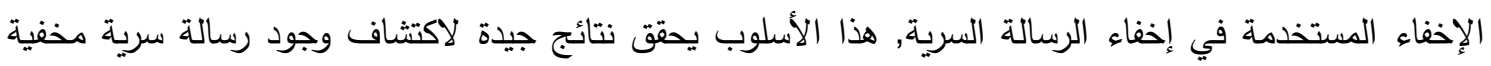

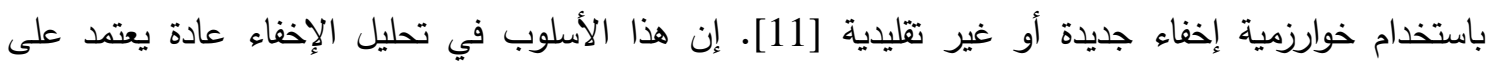

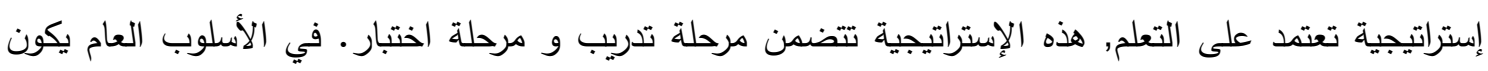

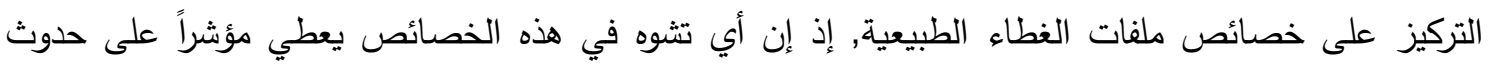
تلاعب بالغطاء مما يعني احتمال وجود رسالة مخفية. تقنيات تحليل الإخفاء للصور في كلتا الحالتين المحدد والعام تصمم غالباً لاكتشاف وجود وسئه أو عدم وجود

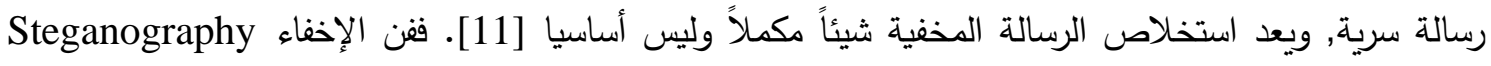

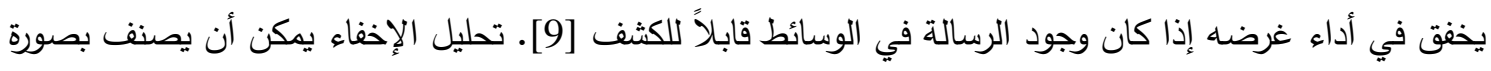
عامة إلى صنفين [3][8][13]

\section{Passive Steganalysis أ- تحليل سلبي للإخفاء}

$$
\text { تحديد فيما إذا كان الملف يحوي بيانات مخفية أم لا. }
$$

\section{بctive Steganalysis تحليل فعال للإخفاء}

$$
\text { تخمين طول الرسالة وموقعها. }
$$$$
\text { تخمين المفتاح السري المستخدم في التضمين. }
$$

تخمين بعض معاملات خوارزمية الإخفاء المستخدمة.

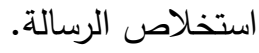

تحليل الإخفاء مجال بحثي جديد نسبياً يهدف إلى كثف و/أو تخمين المعلومات المخفية بمعرفة معلومات قليلة أو بدون معرفة أية معلومات حول خوارزمية الإخفاء المستخدمة أو معاملاتها [2]. قلة المعلومات المتوفرة حول الملف المشكوك فيه يولد الكثير من التساؤلات والتحديات. وهناك على الأقل خمسة تحديات تتعلق بالمعرفة

حول الملف المشكوك فيه تواجه تحليل الإخفاء [5]: أ- الملف المشكوك فيه ربما يحوي أو قد لا يحوي بيانات مخفية. ب- البيانات المخفية قد تكون أو قد لا تكون مشفرة قبل تضمينها في الوسط المضيف. 
ج- الملف المشكوك فيه ربما يحوي ضوضاء أو قد يكون خالياً من الضوضاء. دـ قد يكون بالإمكان أو ربما يكون من المستحيل استعادة البيانات الدخفية أو استخلاصها. هـ - عملية تحليل الإخفاء تستغرق وقتاً طويلاً.

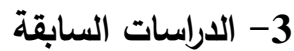

في عام 2003 قدم R. Chadromoulig, Shalin Trivedi طريقة لتحليل الإخفاء إذ استغلا التغيير

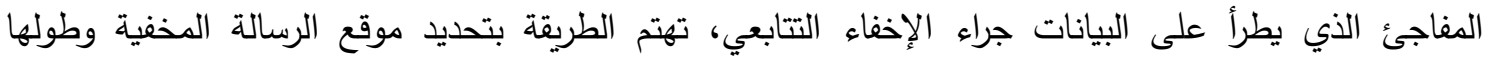

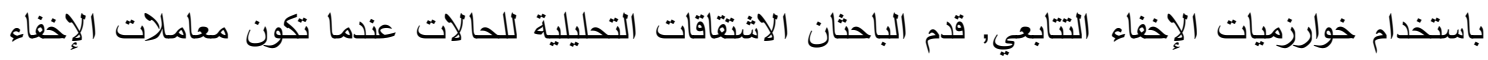

معروفة كلياً أو معروفة جزئياً [11]. في عام 2005 قدم R.Chadromoulig, Shalin Trivedi طريقة كثف تخمن المفتاح السري في

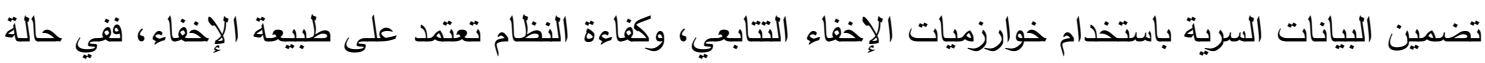
الإخفاء في التردد الواطئ لمعاملات تحويل التجيب المتقطع DCT يكون النظام غير كفوء [18].

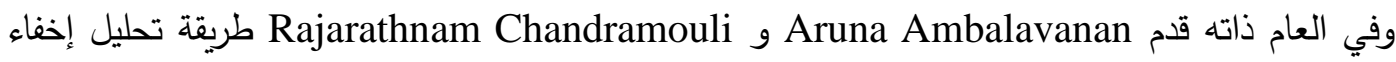
لتخمين الرسالة المخفية بالاعتماد على نظرية باياس Bayes, وذلك بتثكيل الصورة على شكل حقل Markov

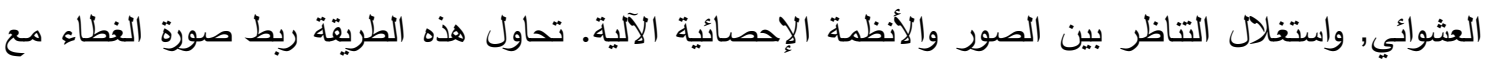
الغطاء المضمن عن طريق دالة احتمالية [1] وفي عام 2010 اقترح M.Revathi وآخرون نظاماً عاماً لاكتثاف وجود الإخفاء وتخمين طول الرسالة

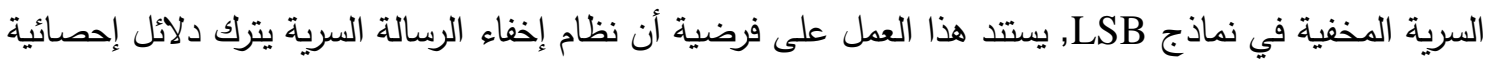

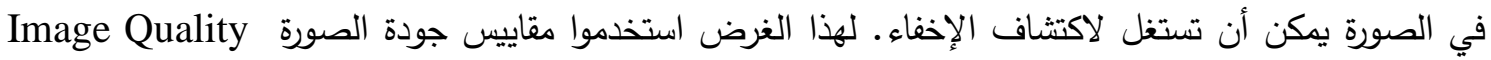
Metric (IQM) وتحليل التباين ANOVA وتحليل الانحدار متعدد المتغيرات بوصفه مصنفاً مثالياً [1]].

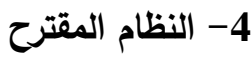

تكتفي معظم بحوث تحليل الإخفاء بدراسة كثف وجود الإخفاء في الوسائط, أو تخمين بعض معاملات

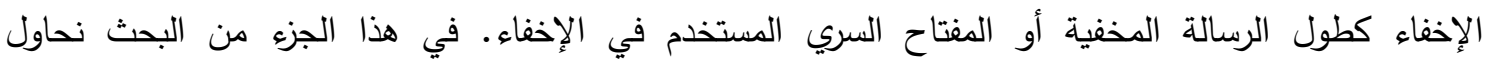

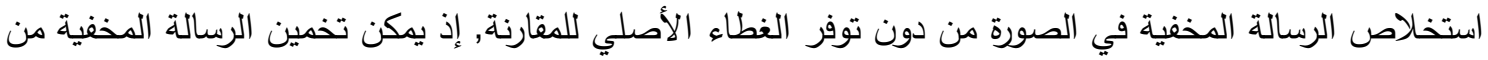
الفرق بين الغطاء والغطاء المضمّن في حال توفر الغطاء. هذا العمل يرتكز على إمكانية تخمين الغطاء الأصلي من الغطاء المضمّن, إذ يكون الإخفاء في نسبة معينة

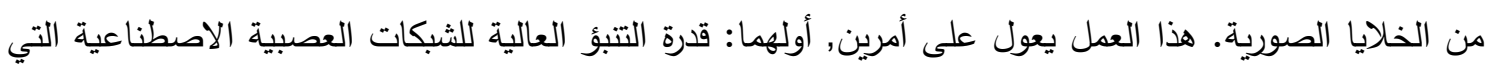

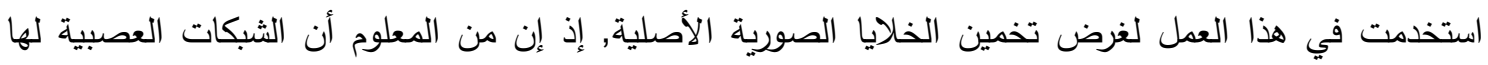

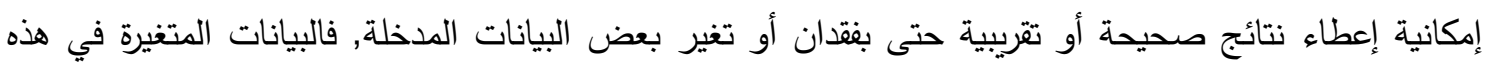

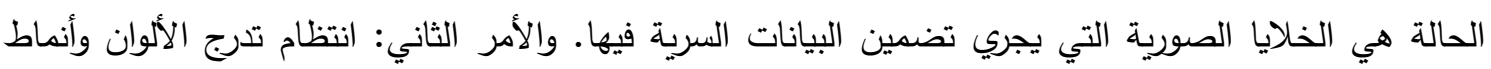
توزيعها في معظم مناطق الصورة, مما يتيح للشبكة العصبية التخمين بدقة عالية. ونظراً لكمية البيانات الهائلة الهتوفرة, يكون من الصعب إيجاد نظام يستطيع تخمين الغطاء الأصلي لكل

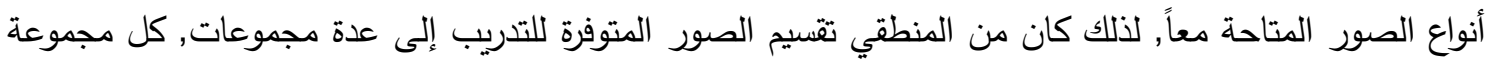


تحوي صوراً متثابهة في مضمونها لتسهيل عملية تخمين الغطاء الأصلي عن طريق توزيع الجهد على أنظمة متعددة كل منها تخمن الصور الأصلية لمجموعة من هذه المجموعات.

أحد أهم التحديات التي تواجه استخلاص البيانات المخفية هو أن البيانات المخفية لا تغير بالضرورة كل هل التهاه الخلايا الثنائية التي تخفى البيانات فيها, إذ لو كانت قيمة الخلية الثنائية المراد إخفاؤها مطابقة لقيمة الخلية الثنائية المضيفة فلن يحصل أي تغيير , مما يجعل من المستحيل كثف هذه الخلية الثنائية دون معرفة مسبقة بمكانها. إن إن

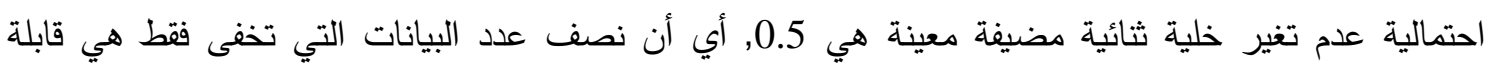

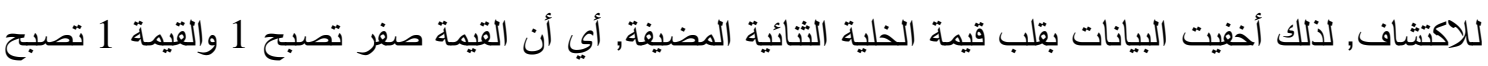

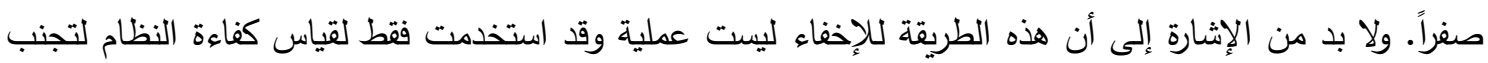
تأثير تغير قيم البيانات الفعلية على أداء النظام. النظام المقترح لاستخلاص البيانات المخفية ينجز على مرحلتين, المرحلة الأولى تدرب فيها شبكة بيرسبترون

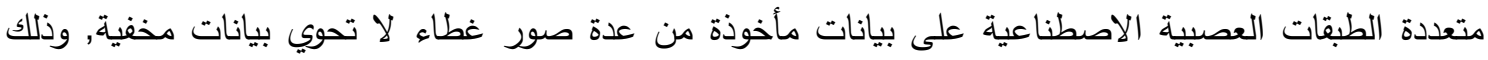

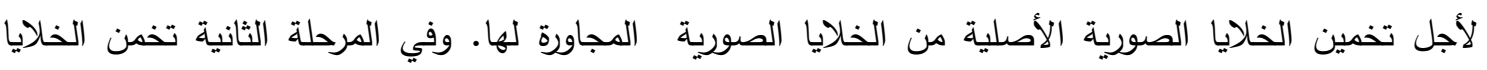
الصورية الأصلية من صور غطاء مضمّن ثم تقارن الخلايا الصورية المخمنة مع الخلايا المقابلة لها في الغطاء المضمّن وتستخلص البيانات المخفية من ناتج المقارنة.

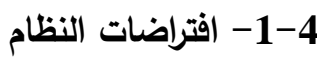
صنفت الصور الرمادية المستخدمة للتدريب والاختبار إلى أربع مجموعات, كل مجموعة تضم صوراً متقاربة في مضمونها (صنفت الصور إلى هذه المجموعات بصرياً), أخفيت البيانات في هذه الصور في الخلية الثنائية الأقل أهمية LSB وأيضاً في مستويات خلايا ثنائية Bit Planes أعلى ( المستوى الثاني والثالث والرابع والخامس) لأجل اختبار مدى قابلية النظام المقترح على استخلاص الرسالة المخفية في هذه المستويات, وكذلك أخفيت البيانات بخمس نسب بداية من نسبة 25\% من سعة الغطاء نزولاً إلى 5\% من سعة الغ الغطاء (يمكن أن يقال أن

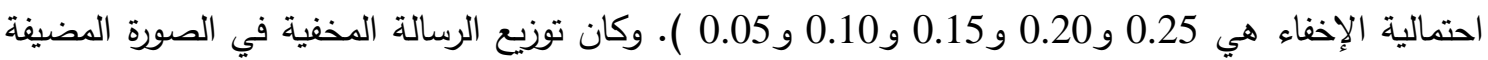
بشكل منتظم لتغطية كامل الصورة. وأخيراً تدرب شبكات مستقلة لكل مجموعة (صنف) من هذه المجاميع.

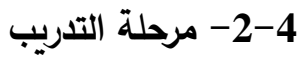

لكي تتمكن الثبكات العصبية من تخمين القيم الأصلية للخلايا الصورية بمعرفة الخلايا الصورية المجاورة لها, لا بد من تدريب هذه الثبكة على بيانات صور غطاء, ويساعد في ذلك كون معظم مناطق الصورة تتألف من

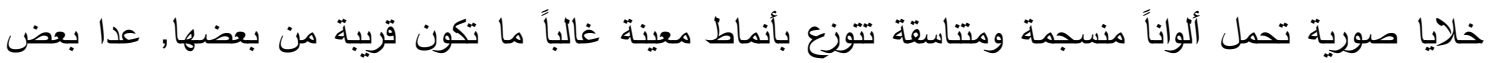

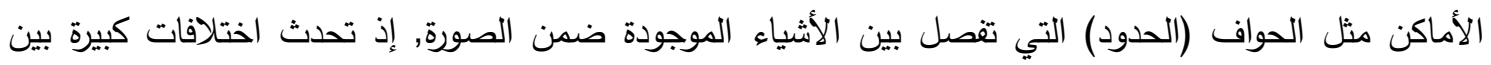

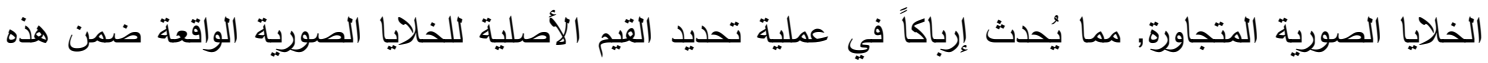

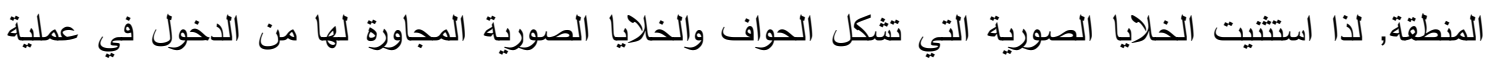
التدريب منعاً لإرباك الثبكة العصبية. 
تبدأ هذه المرحلة بتجميع وتجهيز البيانات لغرض التدريب, ثم إدخال هذه البيانات إلى الثبكة العصبية

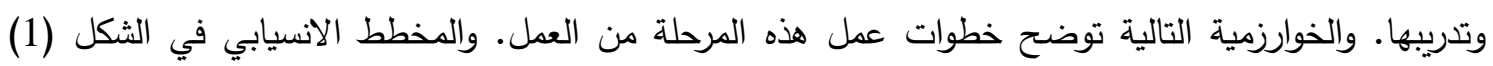
يلخص هذه الخوارزمية. -1-2-4 - خوارزمية مرحلة التدريب

1- قراءة الصور الغطاء وتوسيعها بمقدار خلية واحدة من كل جهة.

2- تحديد حواف الأشياء داخل الصورة وتوسيع الحواف المحددة بعملية Dialation. 3- إضافة الخلايا الصورية غير الواقعة ضمن الحواف الموسعة إلى مصفوفة الهدف t ضمن أزواج التدريب,

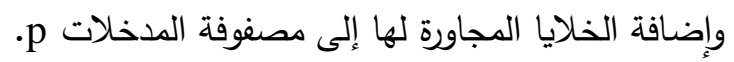
4- تدريب شبكة بيرسبترون متعددة الطبقات باستخدام أزواج التدريب (p, t).

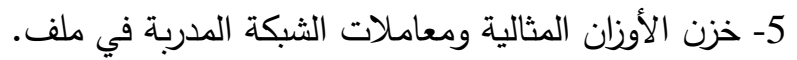

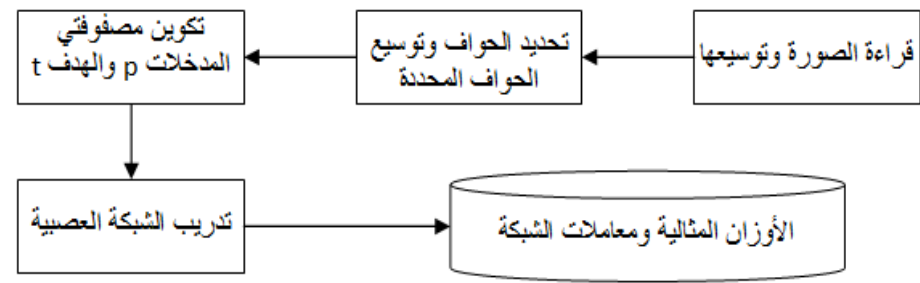

الثكل (1). المخطط الانسيابي لنظام استخلاص البيانات المخفية (مرحلة التدربب).

2-2-4

في هذه المرحلة هيأت صور رمادية نظيفة خالية من الاخفاء, صنفت الصور حسب مضمونها إلى أربع مجموعات, كل منها تحوي 11 صورة متقاربة في مضمونها, الثكل (2) يعرض نماذجاً من الصور من كل هل

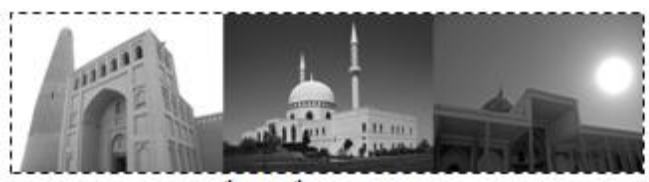

ب. بـ الدجو عة الثاثية.

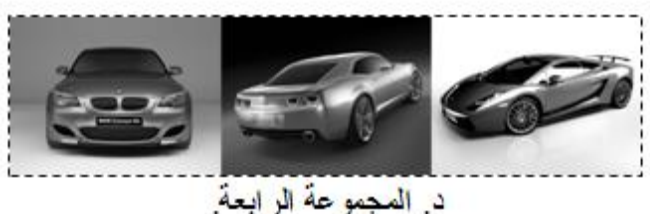

د. - المجمو عة الر ابعة.

الثكل (2). نماذج من الصور ضمن قاعدة بيانات التدريب. مجموعة من هذه المجموعات.

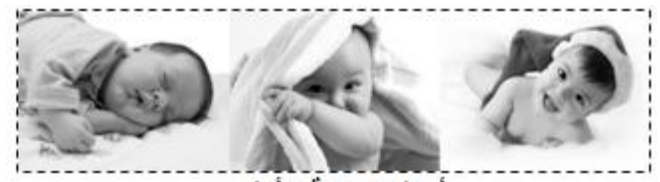

أ. المجموعة الأولى

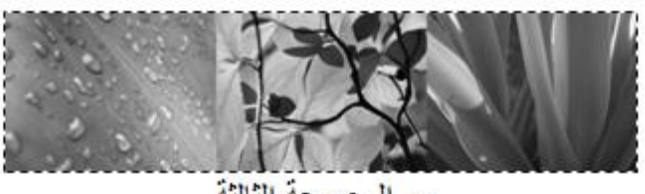

ج. المجمو عة الثنالئم.

3-2-4

بعد قراءة الصورة وخزنها في المصفوفة X, تجري عملية التوسيع لهذه الصورة بمقدار خلية صورية واحدة من

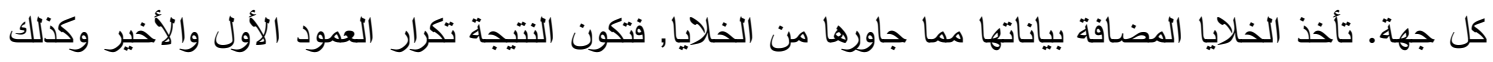
الصف الأول والأخير من الصورة الأصلية, وسبب إجراء هذه العملية هو لكي تدخل الخلايا المتواجدة على أطراف الخاف الصورة الأصلية في المعالجة في الخطوات اللاحقة وتخمن بصورة صحيحة. 
في هذه الخطوة تحدد حواف الأشياء داخل الصورة, بما أن هذه الحواف تمثل منطقة انتقال بين منطقتين كل منها ذات نمط معين لتوزيع الألوان تختلف عن المنطقة الأخرى, فستكون الاختلافات كبيرة بين النقاط المتجاورة.

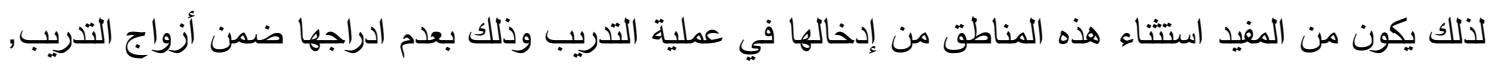
لتجنب إرباك الثبكة العصبية. تحدد الحواف عادة بتنفيذ اللافوف الرياضي بين مصفوفة خلايا الصورة ومرشح المرور العالي. وتحديد الحواف يعتمد على تقريب متقطع لعملية الاثتقاق, وعملية الاشتقاق تقيس معدل التغير في الدالة (الإضاءة في لتئي هذه الحالة). والتغير الكبير في إضاءة الصورة ضمن حيز مكاني صغير يدل على وجود حافة [19]. يستخدم عامل برويت Prewitt Operator لأجل تحديد الحواف في هذا العمل. اختير مرشح برويت لكونه يناسب الصور ذات التباين العالي [19]. يبحث عامل برويت لتحديد الحواف عن الحواف بالاتجاهين الأفقي X والعمودي Yث تدمج هذه المعلومات للحصول على حواف موحدة. ويعتمد على مرشحين أحدهما للاتجاه الأفقي والآخر للاتجاه العمودي. تجري ولاتي عملية

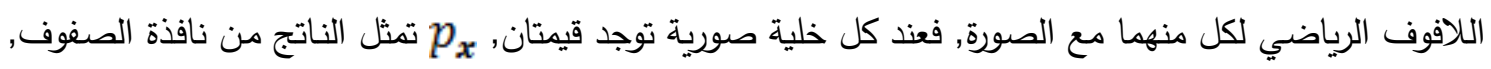

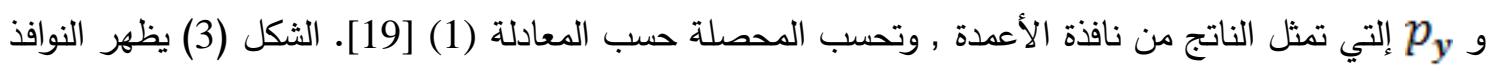
المستخدمة في حساب قيمة الانحدار باتجاه X و Y و والثكل (4)(ب) يظهر مثالاً على تحديد الحواف للصورة

$$
\begin{aligned}
& {\left[\begin{array}{ccc}
-1 & 0 & 1 \\
-1 & 0 & 1 \\
-1 & 0 & 1
\end{array}\right] \quad\left[\begin{array}{ccc}
-1 & -1 & -1 \\
0 & 0 & 0 \\
1 & 1 & 1
\end{array}\right]}
\end{aligned}
$$

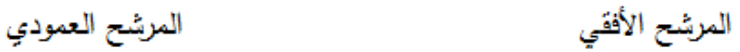

الثكل (3). النوافذ (المرشحات) المستخدمة في حساب قيمة الاحدار.

المعروضة في الثكل (4)(أ).

وتحسب محصلة الاتحدار كالآتي :

$$
p(i, j)=\sqrt{p_{x}^{2}(i, j)+p_{y}^{2}(i, j)} \cdots(1)
$$

إذ إن : -

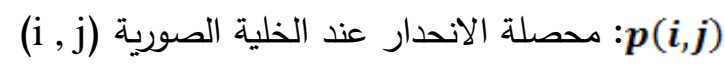

$$
\begin{aligned}
& \text { X تمثل قيمة الانحدار باتجاه }
\end{aligned}
$$$$
\boldsymbol{p}_{\boldsymbol{x}}(\mathrm{x}, \mathrm{y})=[\mathrm{f}(\mathrm{x}-1, \mathrm{y}-1)+\mathrm{f}(\mathrm{x}, \mathrm{y}-1)+\mathrm{f}(\mathrm{x}+1, \mathrm{y}-1)]
$$$$
-[f(x-1, y+1)+f(x, y+1)+f(x+1, y+1)]
$$

$$
\begin{aligned}
\boldsymbol{p}_{\boldsymbol{y}}(\mathrm{x}, \mathrm{y})= & {[\mathrm{f}(\mathrm{x}-1, \mathrm{y}-1)+\mathrm{f}(\mathrm{x}-1, \mathrm{y})+\mathrm{f}(\mathrm{x}-1, \mathrm{y}+1)] } \\
& -[\mathrm{f}(\mathrm{x}+1, \mathrm{y}-1)+\mathrm{f}(\mathrm{x}+1, \mathrm{y})+\mathrm{f}(\mathrm{x}+1, \mathrm{y}+1)]
\end{aligned}
$$

$$
\text { هي الصورة عند النقطة (x, f(x, y) }
$$




\section{- 4}

في هذه الخطوة توسع الحواف المحددة من الخطوة السـابقة, إذ أن معظم هذه الحواف هي بسمك خلية صورية واحدة, لكن منطقة الاختلافات اللونية الكبيرة بين الخلايا المتجاورة ضمن الحدود الفاصلة بين شيئين داخل الصورة تكون أوسع من مجرد خلية صورية واحدة؛ لذا من المفيد توسيع منطقة الاستثناء من الدخول في عملية

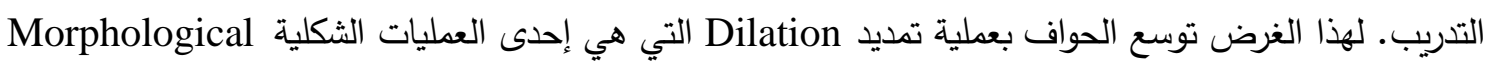

.Operations

تجرى عملية التمديد بإسقاط نافذة صغيرة الحجم (تمثل العنصر التركيبي للثكل) على الصورة وتزحيفها عبر الصورة بأسلوب مشابه للافوف الرياضي تسمى عملية تزحيف النافذة. يتم اختيار الخلية الصورية المطابقة للخلية

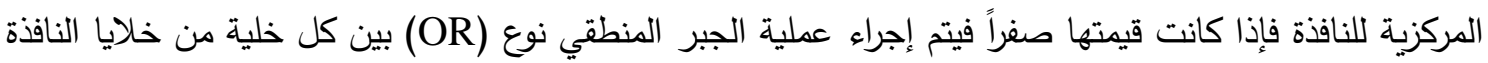
مع الخلايا الصورية المطابقة لها, أما إذا كانت قيمتها تساوي 1 فلا تجرى عملية الجبر المنطقي وتزحف النافذة بمسافة خلية صورية واحدة [19]. الثكل (4)(ج) يظهر مثالاً على توسيع الحواف المحددة. والثكل (4)(د) يبين

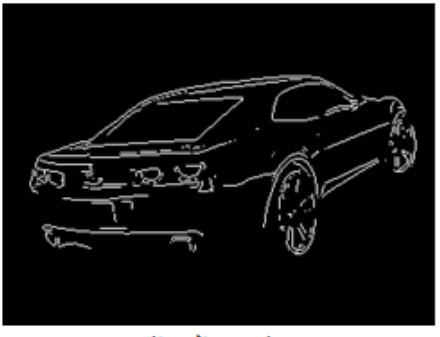

ب. تحبِ الحو افت.

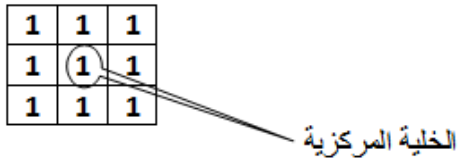

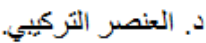
العنصر التركيبي المستخدم لإجراء عملية التمديد في هذا العمل.

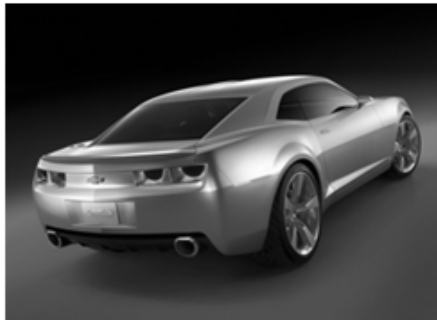

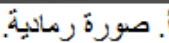

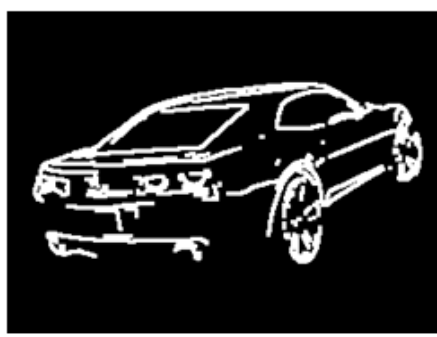

ج. الحو اف الموسعة.

الثكل (4). نموذج لتحديد الحواف وتوسيعها.

-6-2-4 -6 تجهيز بيانات التدريب (المدخلات والههف)

تجز في هذه الخطوة مصفوفتان, مصفوفة المدخلات p بحجم (x) إن n هو عدد الخلايا الصورية في قاعدة بيانات التدريب. تجهز بيانات هاتين المصفوفتين بتزحيف نافذة بحجم 3×3 على الصورة الرمادية الموسعة. إذا كانت قيمة الخلية الصورية لمصفوفة الحواف الموسعة المقابلة للخلية المركزية للنافذة تساوي صفراً فسوف تعطى قيمة الخلية الصورية للصورة الرمادية الموسعة المقابلة للخلية المركزية للنافذة إلى العنصر i من مصفوفة الهدف t, وأيضاً تعطى قيم الخلايا الصورية للصورة الرمادية الموسعة المقابلة لبقية خلايا النافذة إلى العمود i من مصفوفة المدخلات p وحسب التسلسل الموضح في الثكل (5), أما إذا كانت قيمة الخلية الصورية لمصفوفة الحواف الموسعة المقابلة للخلية المركزية للنافذة تساوي 1 فتُهمل بيانات الصورة 
الرمادية الموسعة المقابلة للنافذة. ثم تزحف النافذة بمسافة خلية واحدة وتكرر العملية لتشمل جميع الخلايا الصورية ضمن قاعدة بيانات التدريب.

\begin{tabular}{|c|c|c|}
\hline $\mathrm{P}_{1 \mathrm{i}}$ & $\mathrm{P}_{2 \mathrm{i}}$ & $\mathrm{P}_{3 \mathrm{i}}$ \\
\hline $\mathrm{P}_{4 \mathrm{i}}$ & $\mathrm{t}_{\mathrm{i}}$ & $\mathrm{P}_{5 \mathrm{i}}$ \\
\hline $\mathrm{P}_{6 \mathrm{i}}$ & $\mathrm{P}_{7 \mathrm{i}}$ & $\mathrm{P}_{8 \mathrm{i}}$ \\
\hline
\end{tabular}

الثكل (5). تسلسل توزيع الخلايا الصورية المقابلة للنافذة على مصفوفتي بيانات التدريب ضمن العمود i.

2-4-2-4 تدربب الشبكة العصبية

استخدمت شبكة بيرسبترون متعددة الطبقات Multilayer Perceptron MLP في النظام المقترح لأجل تخمين خلية صورية معينة مما جاورها من خلايا صورية, وذلك بتلقيمها الخلايا الصورية المجاورة للخلية المراد

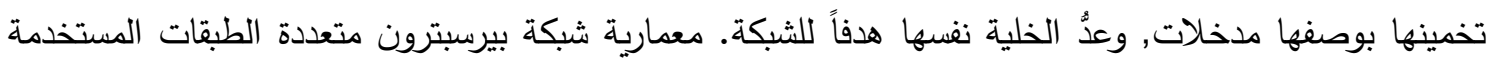
في هذا العمل موضحة في الثكل (6). طبقة الإدخال تتكون من ثمان عقد إذ تستقبل أعمدة المصفوفة P بوصفها مدخلات, في حين أن الطبقة المخفية الوحيدة تحتوي على عشر عقد, أما طبقة الإخراج فتتكون من عقدة وحيدة مخرجاتها أعداد صحيحة قيمة إخراجها

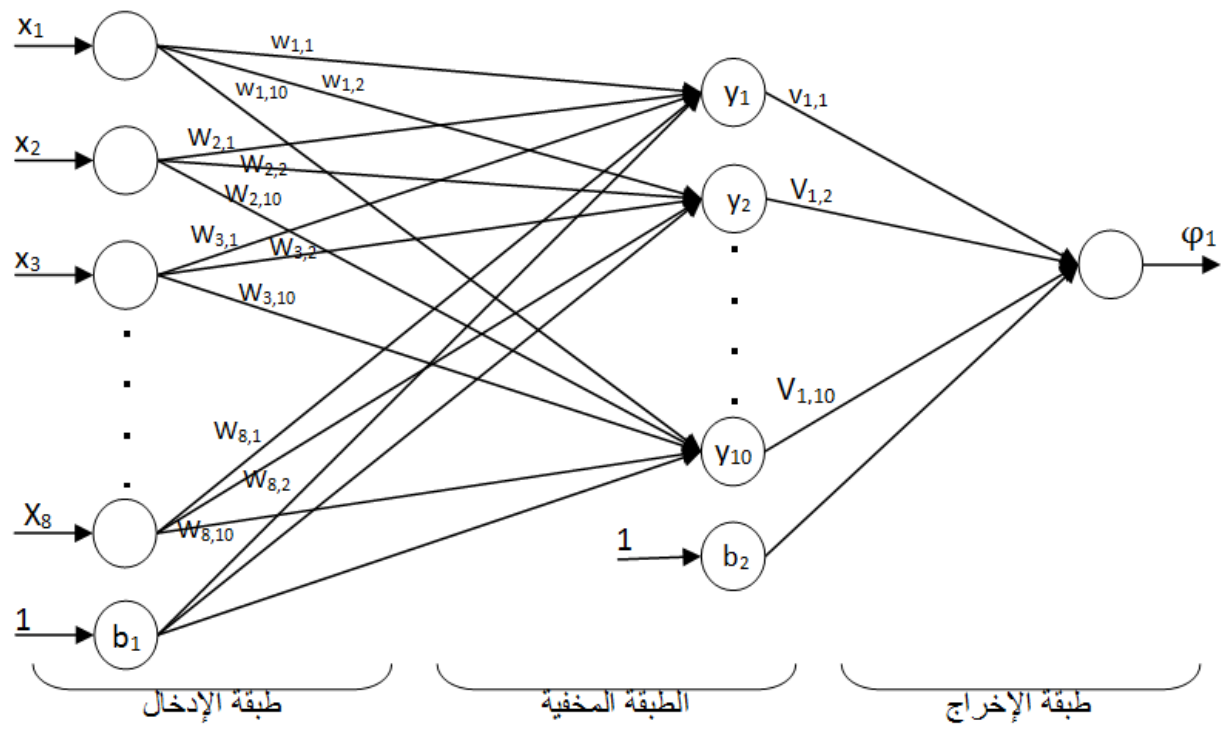

الثكل (6). معمارية شبكة بيرسبترون متعددة الطبقات المستخدمة في النظام المقترح.

المستهدف يتراوح من صفر إلى 255 التي تمثل قيمة الخلية الصورية المراد تخمينها. تدرب الثبكة باستخدام عدد من أزواج التدريب (الإدخال والهدف), إذ استخدمت خوارزمية الانتشار العكسي للخطأ باستخدام قاعدة التدريب Gradient descent with momentum وقد حددت نسبة التعلم بالقيمة 0.01 والزخم بالقيمة 0.9. بعد إتمام التدريب تحفظ أوزان ومعاملات الشبكة المدربة في ملف لغرض استخدامها لتخمين الخلايا الصورية فيما بعد. -3-4 - 3رحلة التخمين بعد إجراء عملية تدريب الشبكة العصبية والحصول على الأوزان المثالية, تستخدم هذه الثبكة بوضعها المثالي لأجل تخمين القيم الأصلية للخلايا الصورية للغطاء المضمّن. يستخدم في هذا العمل صور غطاء مضدّن أخفيت فيها البيانات في الخلايا الثنائية من عدة مستويات, ابتداءً من المستوى الأول الأقل أهمية وإلى المستوى لإنياء 
الخامس وأخفيت البيانات في هذه المستويات بنسب مختلفة من إجمالي سعة الغطاء, ابتداءً من 25\% إلى 5\%

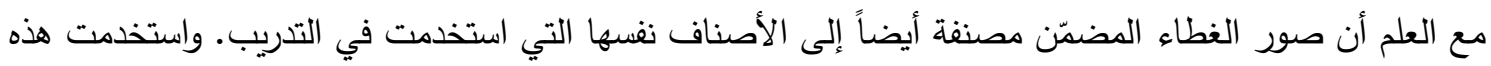
التشكيلة لأجل بيان أداء النظام في هذه الحالات وكذلك بيان تأثير نسبة الإخفاء وكذلك مستوى الإخفاء على أداء النظام المقترح.

تبدأ مرحلة التخمين بتجميع بيانات الاختبار وتجهيزها, ثم إدخال هذه البيانات إلى الثبكة العصبية الاصطناعية المدربة للحصول على الناتج الذي يمثل تخميناً للقيمة الأصلية للخلية الصورية, ثم تقارن هذه النتائج

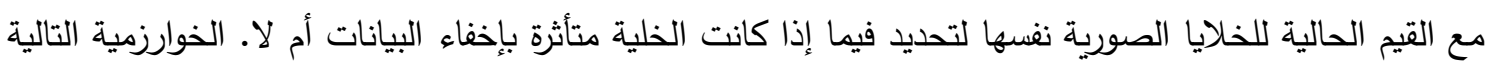
توضح خطوات عمل النظام في مرحلة التخمين, والمخطط الانسيابي في الثكل (7) يلخص هذه الخوارزمية. -4-3-4 - خوارزمية مرحلة التخمين

1- قراءة محتويات الصورة (الغطاء المضمّن) وتوسيعها بمقدار خلية واحدة من كل جهة.

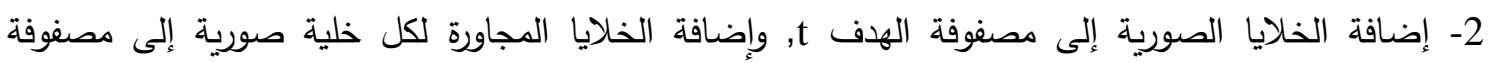
الدخلات p في عمود المقابل لتلك الخلية.

3- إجراء عملية التخمين باستخدام الثبكة العصبية المدربة ومصفوفة المدخلات p, وخزن الناتج في المصفوفة y y. y. 4- قارنة الناتج y مع مصفوفة الهدف t وإجراء عملية تعتيب لناتج المقارنة للحصول على سلسلة ثنائية تمثل

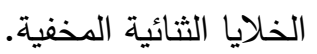

\section{-2-3-4- تهيئة مدخلات الشبكة}

تقرأ الصور الغطاء المضمّن المراد استخلاص البيانات المخفية منها وتخزن في المصفوفة X, ثم تجرى عليها عملية توسيع كما في الفقرة (4-3-3). ثم تجهز مصفوفتان, مصفوفة المدخلات p بحجم (8×n) ومصفوفة

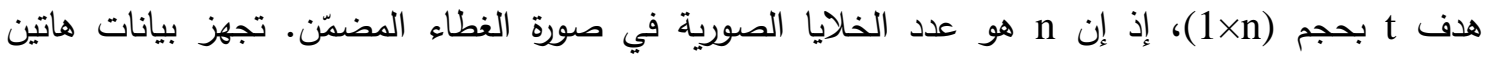
المصفوفتين بتمرير نافذة بحجم 3×3 على المصفوفة x بعد التوسيع, إذ تعطى قيمة الخلية للمصفوفة الموسعة

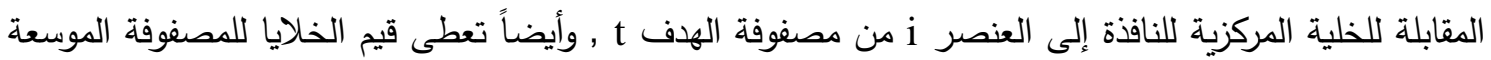

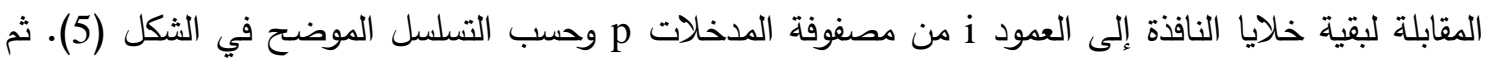
تزحف النافذة بمسافة خلية واحدة وتكرر العملية لتثمل جميع الخلايا الصورية ضمن الصور الغطاء المضمّن المراد استخلاص البيانات المخفية منها.

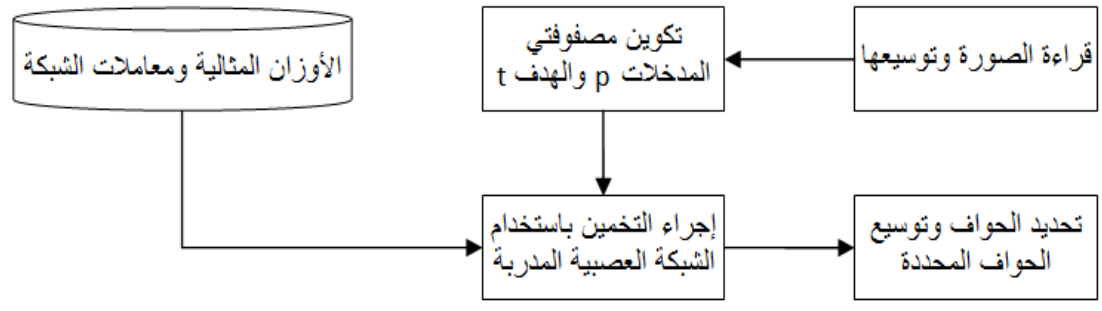

الثكل (7). المخطط الانسيابي لنظام استخلاص البيانات المخفية (مرحلة التخمين). 
-3-3-4 إجراء التخمين باستخدام الثبكة العصبية

تخمن الخلايا الصورية باستخدام شبكة الانتشار العكسي العصبية المدربة في المرحلة السابقة, وذلك بتلقيمها عموداً من مصفوفة المدخلات p المهيأة من الخطوة السابقة (الفقرة 4-4-2), عندها تحسب مخرجات الطبقة $H O_{j}=f\left(\left(\sum_{i=1}^{8} I_{i} * w_{i j}\right)+w_{0 j}\right) \cdots(2)$

المخفية للشبكة العصبية كما في المعادلة (2). إذ إن: ز: تسلسل العقدة في الطبقة المخفية, تتراوح من 1 إلى 10. HO .p ili W: أوزان المدخلات إلى الطبقة المخفية.

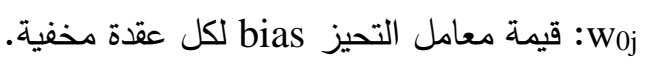
ثم تحسب مخرجات طبقة الإخراج للشبكة (أي الناتج النهائي للشبكة) كما في المعادلة (3). $y=f\left(\left(\sum_{j=1}^{10} H O_{j} * w_{j}\right)+v_{0}\right)$

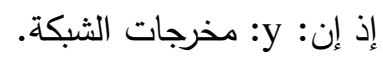

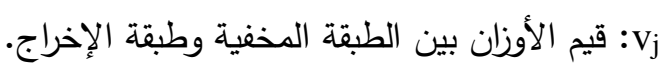
bias لطبقة الإخراج. هذا الناتج يمثل القيمة الأصلية المخمنة للخلية الصورية المراد تخمين قيمتها الأصلية, وهكذا تكرر العملية لتخمين القيم الأصلية لجميع الخلايا الصورية. 4-4-4-4 استخلاص البيانات المخفية بعد الحصول على القيم المخمنة للخلايا الصورية y تقارن مع القيم الحالية لهذه الخلايا الصورية التي وضعت في t, ثم نجرى عملية تعتيب لمطلق الفرق بين القيمتين و كما موضح في المعادلة (4). $e=\left\{\begin{array}{ll}0 & \text { if }|y-t|<\theta \\ 1 & \text { if }|y-t| \geq \theta\end{array} \quad \cdots(4)\right.$

إذ إن: 0: قيمة العتبة. e : مؤشر تغير الخلية الصورية. فإذا كانت قيمة مطلق الفرق بين القيمتين دون العتبة فهذا يعني أن الخلية الصورية لم تتأثر بتضمين

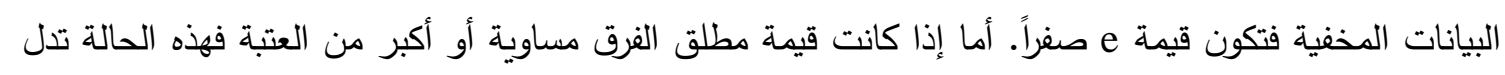
على أن الخلية الصورية قد تأثرت بتضمين البيانات, ما يستتتج أنها خلية مضيفة للبيانات المخفية.

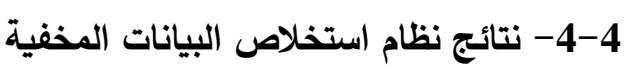
درب نظام استخلاص البيانات المخفية باستخدام بيانات التدريب ضمن قاعدة البيانات الموضحة في الفقرة

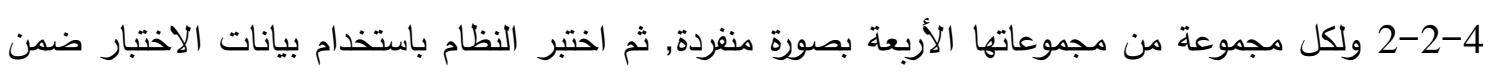

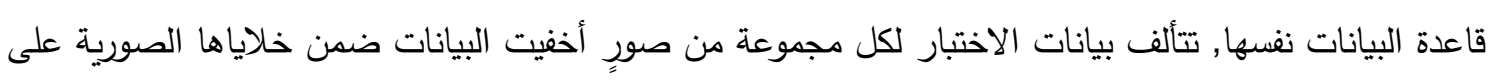

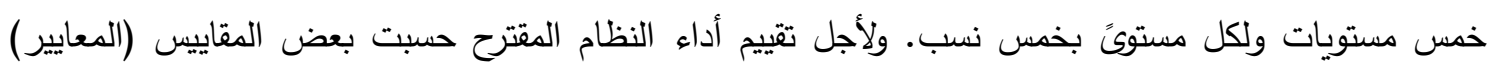


Contingency (وتدعى أيضا بجدول التصادف Confusion Matrix الثائعة اعتماداً على مصفوفة الإرباك (Table), ويمكن تكوين مصفوفة إرباك (Confusion Matrix) بحجم 2×2 لأي مصنف معين ومجموعة من الإبكاك الأمثلة ( مجموعة اختبار) لتمثيل طبيعة أمثلة المجموعة. هذه المصفوفة تمثل الأساس للعديد من المعايير الثائعة. شكل (8) يظهر مصفوفة الإرباك. الأرقام على طول المحور الرئيسي للمصفوفة تمثل القرارات الصائبة

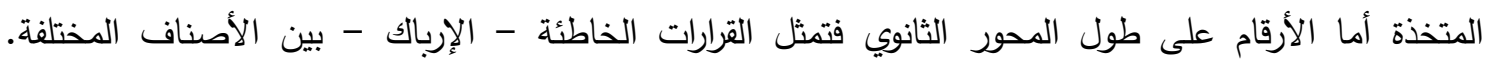
والمعادلات (5) ... (8) هي لبعض المعايير الثائعة التي يمكن حسابها من مصفوفة الإرباك [6]. الأصناف الفعلية

$\mathrm{p} \quad \mathrm{n}$

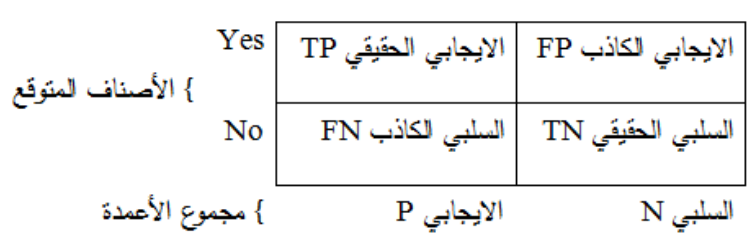

شكل (8). مصفوفة الإبراك.

FPrate $=\frac{F P}{N} \quad \cdots \quad(5)$

TPrate $=\frac{T P}{P} \cdots(6)$

precision $=\frac{T P}{T P+F P} \cdots(7)$

accuracy $=\frac{\mathrm{TP}+\mathrm{TN}}{\mathrm{P}+\mathrm{N}} \cdots(8)$

إذ أن: Tprate: معدل الإيجابي الحقيقي (أيضا يدعى بنسبة معدل الإصابة والتذكر ) للمصنف, وكذلك يشار إليه بمصطلح التذكر Recall, وأيضاً الحساسية Sensitivity. Fprate Positive Predictive Value القيمة التنبؤية الإيجابية Precision Accuracy المخططات البيانية لمقياس الدقة Accuracy للمجموعات الأربعة بمختلف النسب والمستويات مبينة في الثكل (9)-الثكل (12). حسب مقياس الدقة وفقاً للمعادلة (8). من أجل تحليل نتائج اختبار النظام المقترح, أخذ معامل

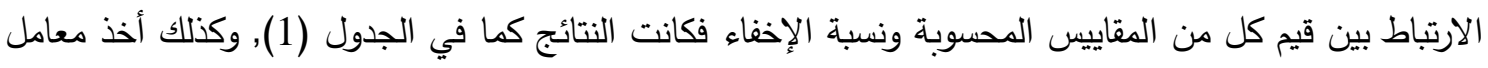

الارتباط بين هذه المقاييس ومستوى الخلايا الثنائية الذي تخفى البيانات فيه فكانت النتائج كما في الجدول (2). الجدول (1). معامل الارتباط مع نسبة الإخفاء.

\begin{tabular}{|c|c|c|c|c|}
\hline Accuracy & Precision & TP rate & FP rate & \\
\hline-0.97671 & -0.26098 & -0.75643 & 0.630234 & المجموعة 1 \\
\hline-0.99384 & 0.030304 & -0.7548 & 0.736769 & المجموعة 2 \\
\hline-0.99616 & 0.271388 & -0.7657 & 0.825747 & المجموعة 3 \\
\hline-0.9301 & -0.28957 & -0.79892 & 0.669416 & المجموعة 4 \\
\hline-0.9742 & -0.06221 & -0.76897 & 0.715542 & المعدل \\
\hline
\end{tabular}




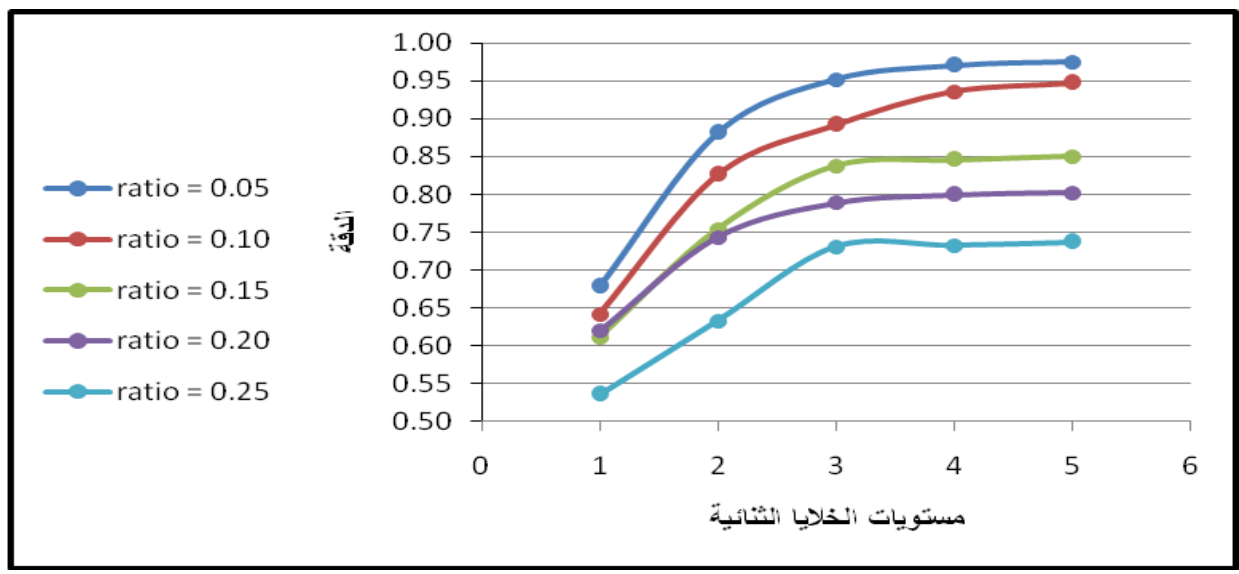

الثكل (9). المخطط البياني لمقياس الدقة Accuracy للمجموعة الأولى.

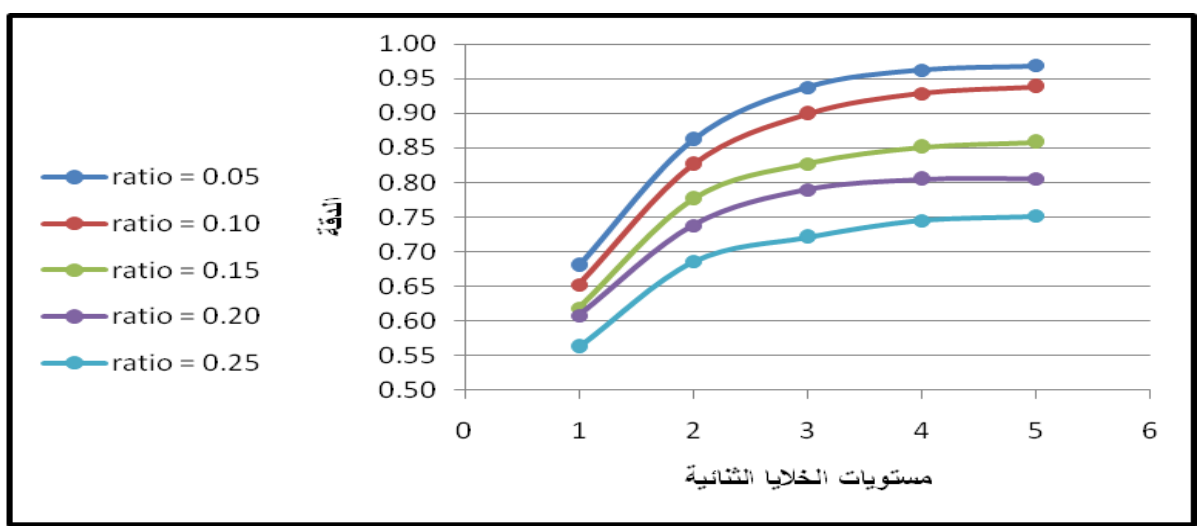

الثكل (10). المخطط البيانى لمقياس الدقة Accuracy للمجموعة الثانية.

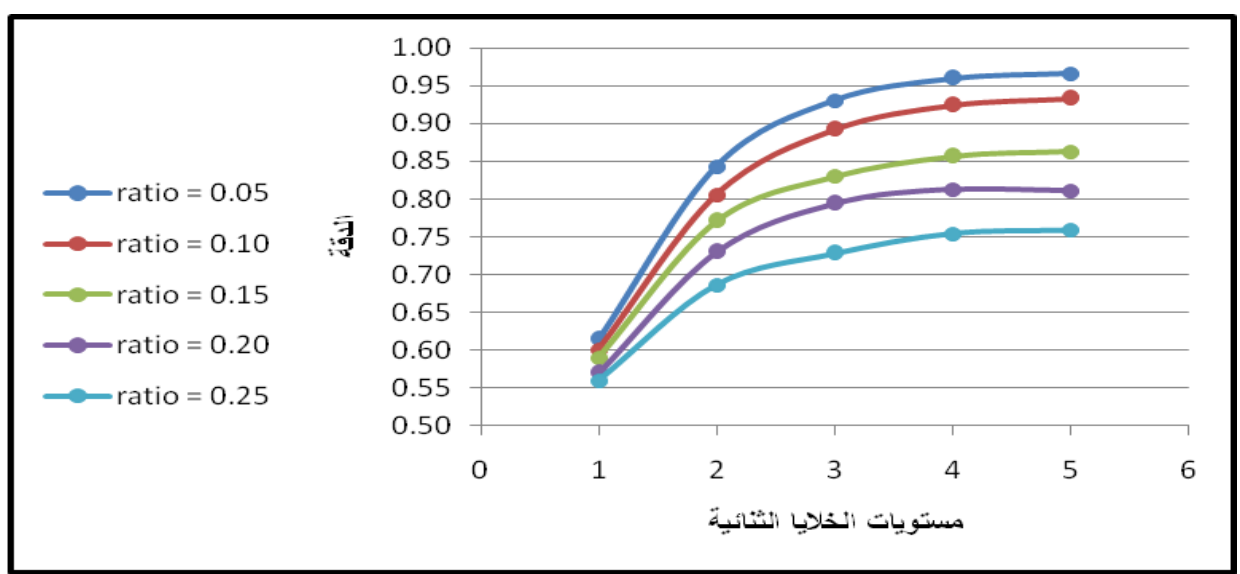

الثكل (11). المخطط البياني لمقياس الدقة Accuracy للمجموعة الثالثة.

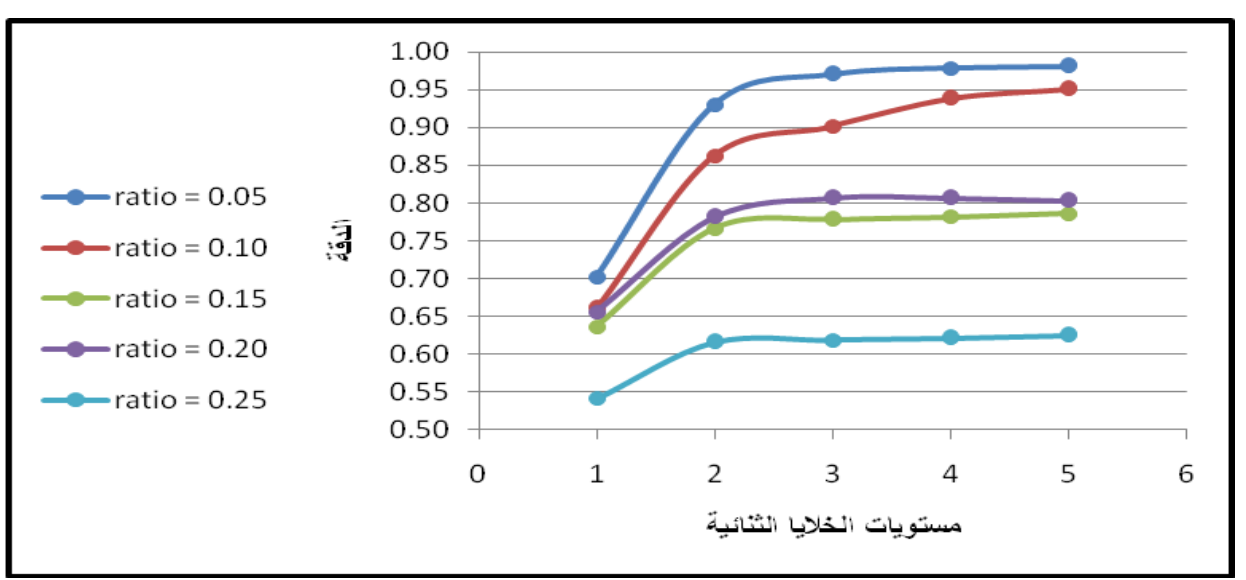

الثكل (12). المخطط البياني لمقياس5الاقة Accuracy للمجموعة الرابعة. 
الجدول (2). معامل الارتباط مع مستوى الخلايا الثنائية.

\begin{tabular}{|c|c|c|c|c|}
\hline Accuracy & Precision & TP rate & FP rate & \\
\hline 0.871624 & 0.795778 & -0.87764 & -0.89303 & المجموعة 1 \\
\hline 0.87813 & 0.990296 & -0.92233 & -0.904 & المجموعة 2 \\
\hline 0.872878 & 0.986652 & -0.94162 & -0.90476 & المجموعة 3 \\
\hline 0.801507 & 0.244966 & -0.88446 & -0.86987 & المجموعة 4 \\
\hline 0.856035 & 0.754423 & -0.90651 & -0.89291 & المعدل \\
\hline
\end{tabular}

نجد أن لمقياس الدقة علاقة عكسية قوية جداً مع نسبة الإخفاء, أي أنها تقل بزيادة نسبة الإخفاء, ولكن

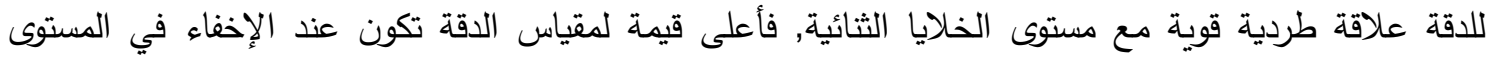

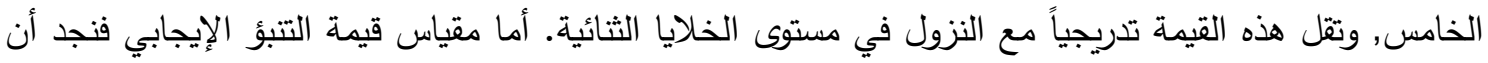

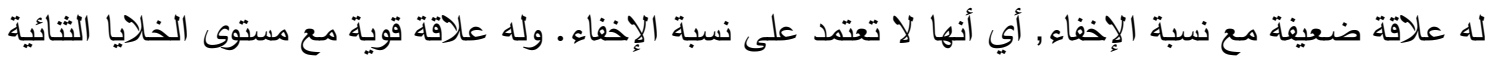
في المجموعات الثثلاثة الأولى, ولكن له علاقة ضعيفة مع مستوى الخلايا الثنائية عند استخدام المجموعة الرابعة,

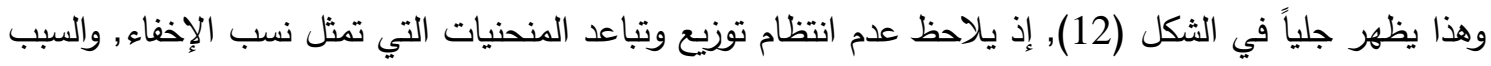
قد يعود إلى طبيعة البيانات في هذه المجموعة.

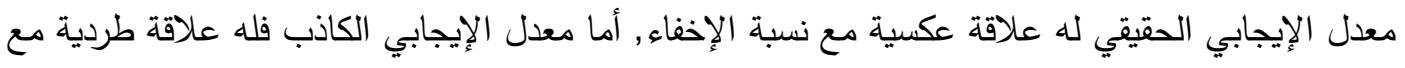

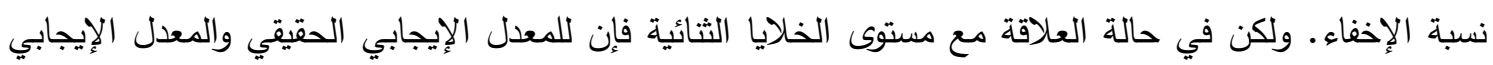
الكاذب علاقة عكسية مع مستوى الخلايا الثنائية. إحدى المشاكل التي تواجه النظام المقترح هي وجود البيانات المتضاربة, والبيانات المتضلاربة تعني أن يكون لمتجهي إدخال متساويين في القيم أهداف مختلفة, أو بتعبير آخر , أن يكون لخليتين صوريتين مختلفتين بالقيم

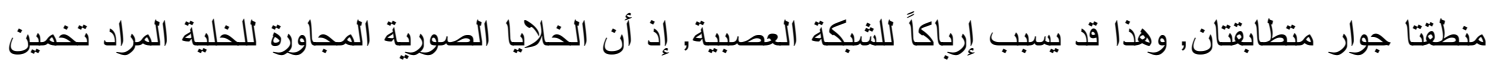
قيمتها تشكل مصفوفة المدخلات p كما موضح في الفقرة 4-2-6. من أجل رصد هذه المشكلة في النظام المقترح حسب عدد حالات البيانات المتضاربة في قاعدة البيانات الخاصة بالنظام المقترح. النسب المئوية للبيانات المتضاربة مبينة في الجدول (3), وهي نسبة البيانات المتضاربة إلى البيانات الكلية. الجدول (3). النسبة المئوية للبيانات المتضاربة.

\begin{tabular}{|c|c|}
\hline النسبة المئوية & \\
\hline $13.53 \%$ & المجموعة 1 \\
\hline $20.28 \%$ & المجموعة 2 \\
\hline $9.10 \%$ & المجموعة 3 \\
\hline $15.53 \%$ & المجموعة 4 \\
\hline
\end{tabular}

طبق النظام المقترح المدرب باستخدام المجموعات الأربعة كلاً على حدة على صورة Lena بحجم 154×1034, الثكل (13), التي استخدمها Aruna Ambalavanan و Rajarathnam Chandramouli لاختبار طريقة باياس لتحليل الإخفاء في الصورة Bayesian Image Steganalysis Approach المقترحة في

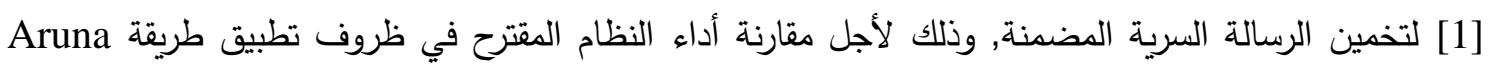


نفسها و Rajarathnam لأنه لم يتسنَ لنا الحصول على البرنامج الخاص بهذه الطريقة لتطبيقها على قاعدة البيانات الخاصة بالنظام المقترح في هذا البحث. اقتصر التطبيق على المستويات الثالث والرابع والخامس لأن نتائج

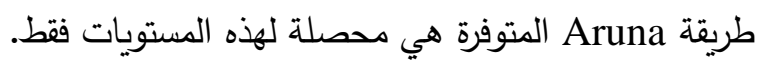

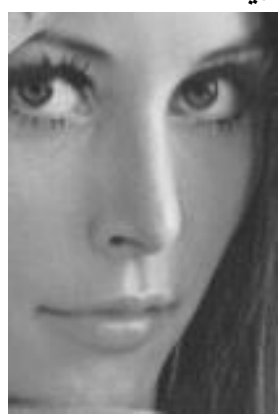

الثكل (13). صورة Lena.

المخططات البيانية لتمثيل الدقة Accuracy للنظام المقترح بالمقارنة مع طريقة Aruna و Rajarathnam

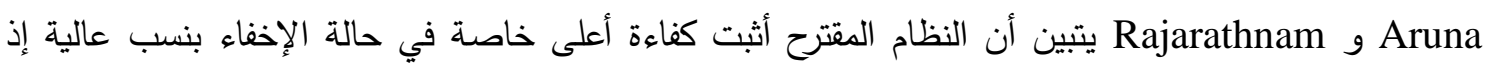
تخفق طريقة Aruna و Rajarathnam. 4-5- الاستنتاجات

انتظام العلاقة ما بين الخلايا الصورية المتجاورة في معظم مناطق الصورة توفر الأرضية المناسبة لبناء نظام لاستخلاص البيانات المخفية في الصور • تنوع البيانات الكبير ضمن قاعدة البيانات المجهزة يتولد عنها

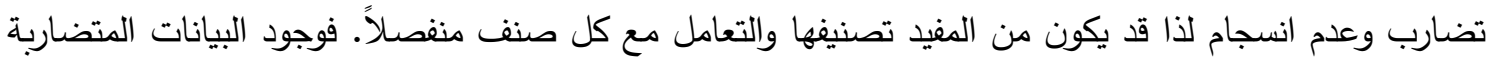
وغير الطبيعية يربك عمل نظام استخلاص البيانات المخفية ويجعل وصوله إلى حالة الاستقرار صعباً. العلاقة بين أداء نظام استخلاص البيانات المخفية ونسبة الإخفاء عكسية, إذ كلما قلت نسبة الإخفاء زادت كفاءة النظام المقترح, وذلك لأن النظام المقترح يعتمد على الخلايا الصورية المجاورة للخلية المراد تخمينها لتخمين قيمتها الأصلية, فكلما زادت نسبة الإخفاء زادت احتمالية تغير قيم الخلايا المجاورة لهذه الخلية مما يؤثر سلباً على لئى

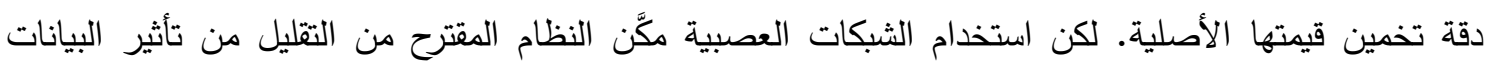

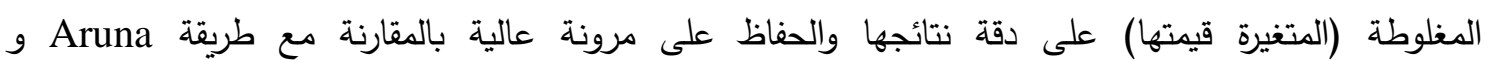
Rajarathnam (المعتمد على صيغ رياضية أقل مرونة), إذ من المعروف أن الشبكات العصبية قادرة على إعطاء نتائج تقريبة حتى بفقدان أو تغير قيم بعض المدخلات. وهذا ييرر تزايد الفرق بين أداء النظامين بزيادة نسبة الإخفاء. العلاقة بين أداء نظام استخلاص البيانات المخفية ومستوى الخلايا الثنائية التي تخفى فيه البيانات علاقة طردية, إذ إن أداء النظام أفضل ما يكون عند المستوى الخامس, في حين يكون الأداء أقل عند المستوى الأول

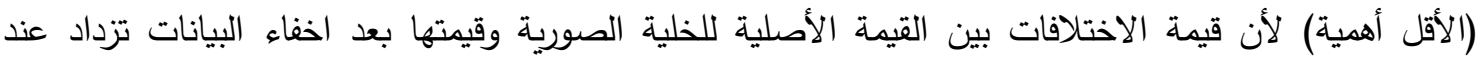




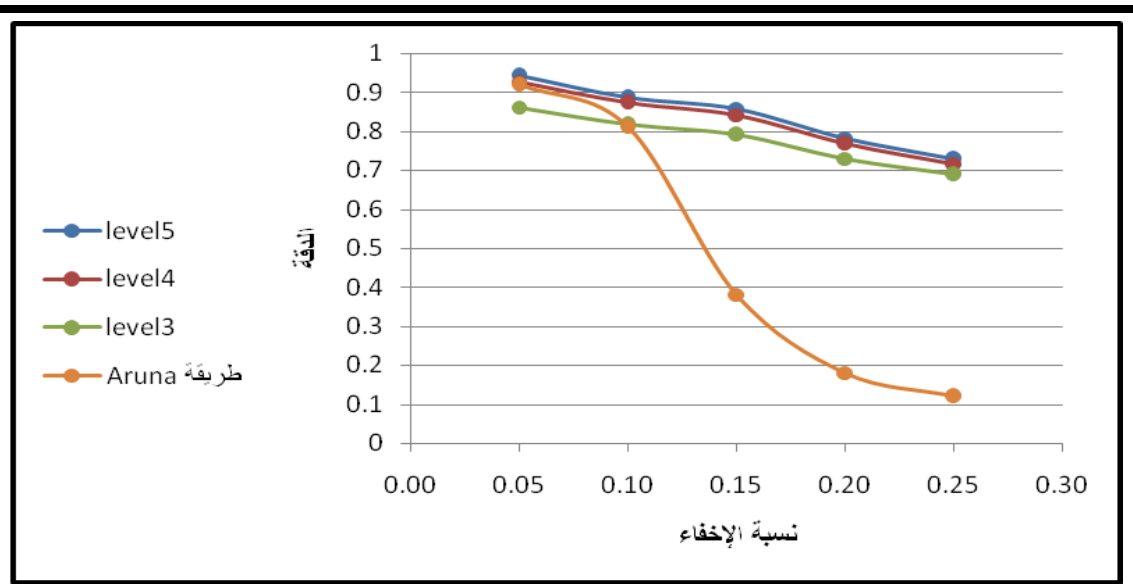

الثكل (14). مخطط بياني لتمثيل دقة النظام المقترح المدرب باستخدام المجموعة الأولى عند تطبيقه على صورة Lena.

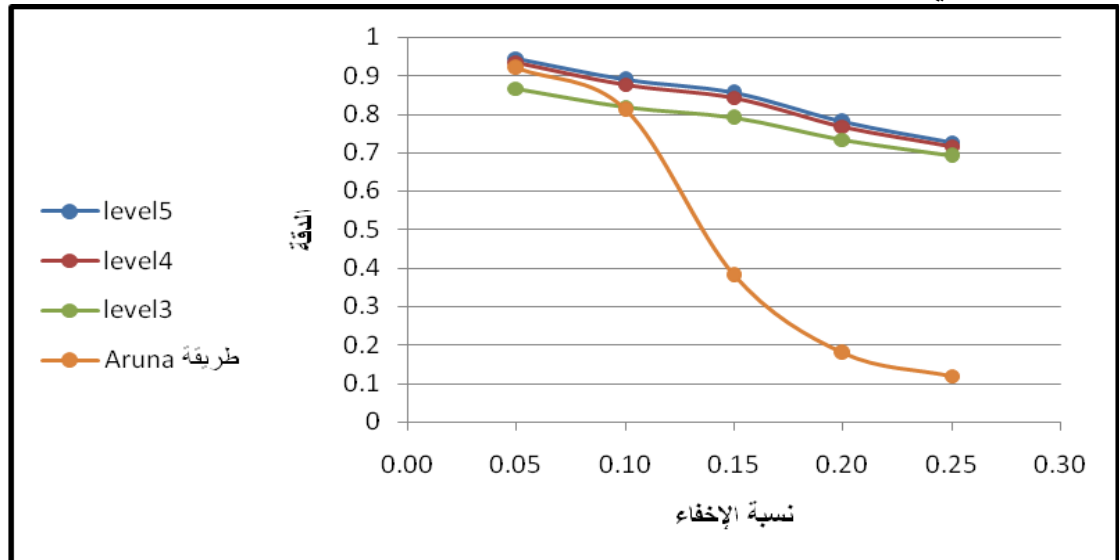

الثكل (15). مخطط بيانى لتمثيل دقة النظام المقترح المدرب باستخام المجموعة الثانية عند تطبيقه على صورة Lena.

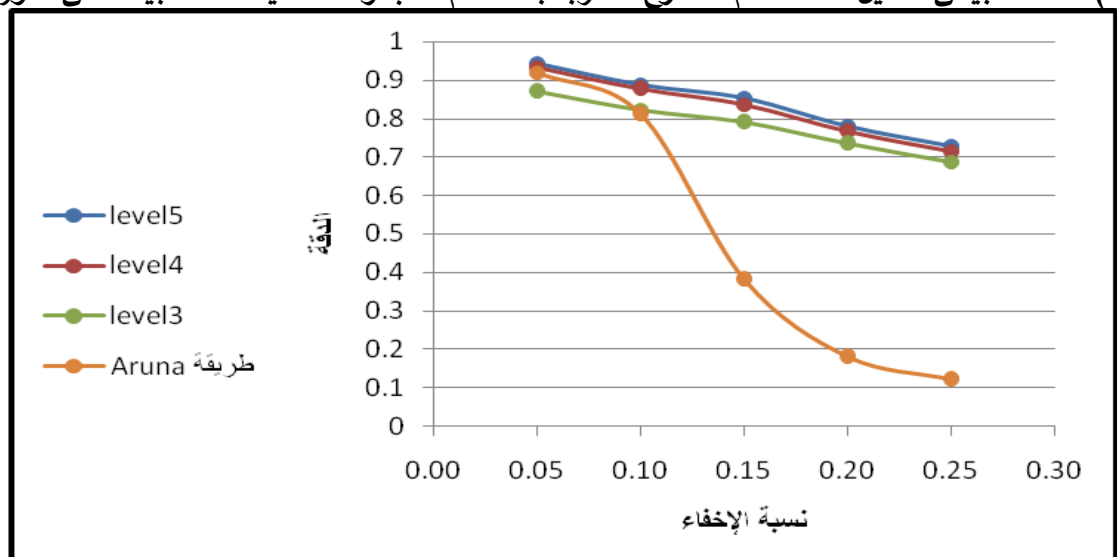

الثكل (16). مخطط بياني لتمثيل دقة النظام المقترح المدرب باستخام المجموعة الثالثة عذ تطبيقه على صورة Lena.

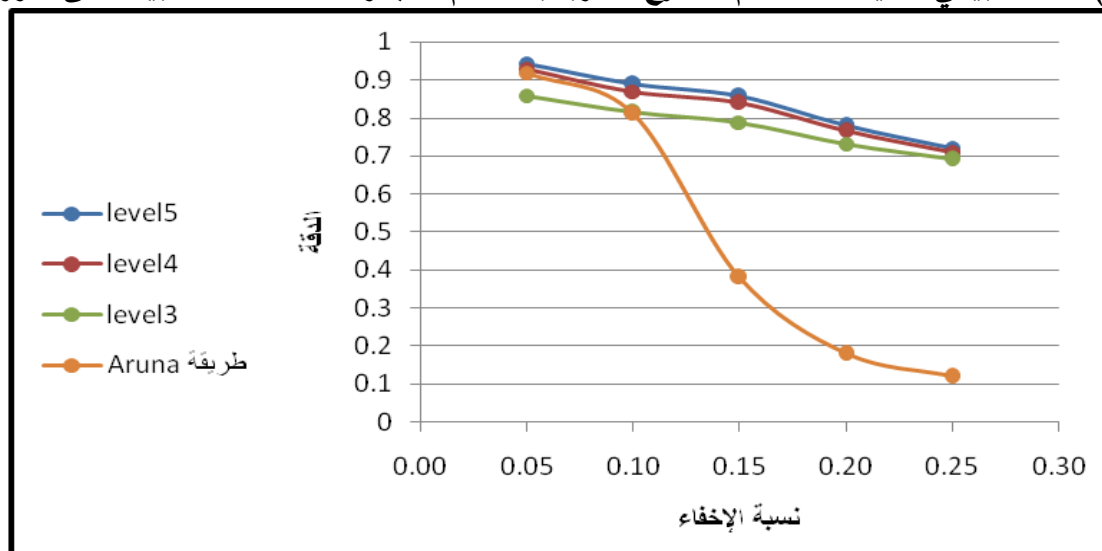

الثكل (17). مخطط بيانى لتمثيل دقة النظام المقترح المدرب باستخدام المجموعة الرابعة عند تطبيقه على صورة Lena. 


\section{المصادر}

[1] Ambalavanan Aruna, Chandramouli Rajarathnam. "A bayesian image steganalysis approach to estimate the embedded secret message". International Multimedia Conference, Proceedings of the Multimedia and Security, ACM Press, New York, USA, pp. 33 - 38, 2005.

[2] Chandramouli R. "A mathematical framework for active steganalysis". ACM Multimedia Systems Journal, Special Issue on Multimedia Watermarking, Multimedia Systems vol. 9, no. 3, pp. 303-311, 2003.

[3] Chandramouli R. and Memon N. D. " Steganography Capacity: A Steganalysis Perspective". SPIE Proceedings of Security and Watermarking of Multimeida Contents, 2003.

[4] Davidson Jennifer, Bergman Clifford and Bartlett Eric. "An Artificial Neural Network for Wavelet Steganalysis". Proceedings of SPIE - The International Society for Optical Engineering, vol. 5916, Mathematical Methods in Pattern and Image Analysis, pp. 1-10, 2005.

[5] Din Roshidi and Samsudin Azman. "Digital Steganalysis: Computational Intelligence Approach". International Journal of Computers, Issue 1, Vol. 3, 2009.

[6] Fawcett Tom. "ROC Graphs: Notes and Practical Considerations for Data Mining Researchers". Technical Report, Intelligent Enterprise Technologies Laboratory, HP Laboratories Palo Alto, HPL-2003-4, Hewlett-Packard Company, January 2003.

[7] Fridrich Jessica and Goljan Miroslav. "Practical Steganalysis-State of the Art". Proc. SPIE Photonics West, Vol. 4675, Electronic Imaging 2002, Security and Watermarking of Multimedia Contents, San Jose, California, pp. 1-13, January 2002.

[8] Kobsi Nouha and Merouani Hayet Farida. "Neural Network Based Image Steganalysis: A Comparative Study". JIG'2007 - 3èmes Journées Internationales sur l'Informatique Graphique. pp. 235-240, 2007.

[9] Lafferty Patricia and Ahmed Farid. "Texture Based Steganalysis: Results for Color Images". Proc. SPIE, vol. 5561, pp. 145-151, Aug 2004.

[10] Li Bin, He Junhui, Huang Jiwu and Shi Yun Qing. "A Survey on Image Steganography and Steganalysis". Journal of Information Hiding and Multimedia Signal Processing, Vol. 2, No. 2, April 2011.

[11] Meghanathan Natarajan end Nayak Lopamudra. "Steganalysis Algorithms for Detecting the Hidden Information in Image, Audio and Video Cover Media". International Journal of Network Security \& Its Application (IJNSA), vol.2, no.1,pp. 43-55, January 2010.

[12] Nosrati Masoud, Karimi Ronak, Nosrati Hamed and Karimi Maryam. "An introduction to steganography methods". World Applied Programming, Vol 1, No 1, pp. 37-41, April 2011.

[13] Özer Hamza. "Audio Watermarking, Steganalysis Using Audio Quality Metrics, and Robust Audio Hashing". Ph.D. thesis, the Institute for Graduate Studies in Science and Engineering, Boğaziçi University, 2005.

[14] Revathi M., Bhattacharjee J. B., Vijayalakshmi S. "Framework of LSB, Adaptive Steganalysis with IQM and Stegnography of Digital Media". Georgian 
Electronic Scientific Journal: Computer Science and Telecommunications, Vol. 24, No.1, pp. 39-48, 2010.

[15] Richer Pierre. "Steganalysis: Detecting Hidden Information with Computer Forensic Analysis". SANS Institute, 2003.

[16] Sabeti Vajiheh, Samavi Shadrokh, Mahdavi Mojtaba and Shirani Shahram. "Steganalysis of Embedding in Difference of Image Pixel Pairs by Neural Network". ISC, vol. 1, no. 1, pp. 17-26, January 2009.

[17] Trivedi Shalin and Chandramouli R. "Active Steganalysis of Sequential Steganography". SPIE conference California, Vol. 5020, No. 13, pp. 123-130, January 2003.

[18] Trivedi Shalin and Chandramouli R. "Secret Key Estimation in Sequential Steganography" IEEE Transactions on Signal Processing, Vol. 53, Issue 2, Part 2, pp. 746-757, Feb 2005.

[19] Umbaugh Scott E. "Computer Vision and Image Processing: A Practical Approach Using CVIP Tools" Prentice Hall RTP, 1998.

[20] Xie Chunhui, Cheng Yimin and Chen Yangkun. "An active steganalysis approach for echo hiding based on Sliding Windowed Cepstrum". Signal Processing, Vol. 91, pp. 87-89, 201. 